\title{
Variational formulation of Eisenhart's unified theory
}

\author{
Nikodem J Popławski \\ Department of Physics, Indiana University, Swain Hall West, 727 East Third Street, \\ Bloomington, IN 47405, USA \\ E-mail: nipoplaw@indiana.edu
}

\begin{abstract}
Eisenhart's unified field theory is based on a non-Riemannian affine connection related to the covariant derivative of the electromagnetic field tensor. The sourceless field equations of this theory arise from vanishing of the symmetrized Ricci tensor and the torsion trace. We formulate Eisenhart's theory from a metric-affine variational principle; a Lagrange multiplier constraining the torsion becomes the source for the Maxwell equations.
\end{abstract}

PACS numbers: 04.20.Fy, 04.50.Kd 


\section{Introduction}

In general relativity, the electromagnetic field and its sources are considered to be on the side of the matter tensor in the Einstein field equations, acting as sources of the gravitational field. The geometry of general relativity is that of a four-dimensional Riemannian manifold, equipped with a symmetric metric-tensor field and an affine connection that is torsionless and metric compatible. In classical unified field theories, the electromagnetic field obtains the same geometrical status as the gravitational field [1]. In order to combine gravitation and electromagnetism on the classical level within a geometrical theory we must modify some postulates of general relativity, resulting in a non-Riemannian geometry [2].

The most known attempts of creating a unified field theory include: Weyl's conformal geometry [3], Kaluza's five-dimensional theory [4], and the Einstein-StrausSchrödinger nonsymmetric field theory [5, 6]. Weyl relaxed the postulate of metric compatibility of the affine connection, obtaining a unified theory, where electromagnetic gauge transformation was related to conformal transformation of the metric. In Kaluza's theory, electromagnetic potentials were represented in a five-dimensional metric, and the Lagrangian proportional to the five-dimensional Ricci scalar yielded the EinsteinMaxwell field equations and the Lorentz equation of motion. Relaxing the postulate of the symmetry of the affine connection [7] and metric tensor resulted in the EinsteinStraus-Schrödinger theory that related the electromagnetic field to the skewsymmetric part of the metric tensor. Although all three theories turned out to be unphysical [8], Weyl's theory introduced the concept of gauge invariance that led to modern particle physics based on non-Abelian SU(n) fields, while Kaluza's theory gave rise to later models with extra dimensions.

Eisenhart showed [9] that one can unify the Maxwell field equations and the Lorentz equation of motion with gravity inside a purely geometrical theory. In Eisenhart's unified field theory, the torsion tensor is related to the Riemannian covariant derivative of the electromagnetic field tensor, and the torsion trace vanishes. This theory was explored further in [10], however, as in [9], the field equations were postulated outside a variational principle. In this paper, we formulate Eisenhart's unified theory as a variational theory. Because the affine connection is independent of the metric, we use the metric-affine formulation of gravity [11, 12, 13, 14], in which both the metric tensor and affine connection are variables (gravitational potentials) with respect to which the total action is varied, and which is dynamically equivalent to the purely metric Einstein-Hilbert formulation [15]. We find and discuss the metric-affine Lagrangian that generates, by varying the connection, Eisenhart's field equations. We include the condition that the torsion trace be zero as a Lagrange-multiplier constraint in the action. This vector turns out to be the source current for the Maxwell equations. 


\section{Eisenhart's unified field theory}

Eisenhart's original theory [9] is based on the affine connection $\Gamma_{\mu \nu}^{\rho}$ that is not metric compatible, depending on the symmetric metric tensor $g_{\mu \nu}$ via the Christoffel symbols $\left\{\begin{array}{c}\rho \\ \mu \nu\end{array}\right\}=\frac{1}{2} g^{\rho \lambda}\left(g_{\nu \lambda, \mu}+g_{\mu \lambda, \nu}-g_{\mu \nu, \lambda}\right)$, and the electromagnetic field tensor $F_{\mu \nu}=A_{\nu, \mu}-A_{\mu, \nu}$ :

$$
\Gamma_{\mu \nu}^{\rho}=\left\{\begin{array}{c}
\rho \\
\mu \nu
\end{array}\right\}+k F_{\mu \nu: \sigma} g^{\rho \sigma},
$$

where the colon denotes the Riemannian covariant differentiation with respect to the Levi-Civita connection $\left\{\begin{array}{c}\rho \\ \mu \nu\end{array}\right\}$, and the physical constant $k$ guarantees the correct dimension $\dagger$ Accordingly, the symmetric part of the connection is equal to the Christoffel symbols, while its skewsymmetric part, the Cartan torsion tensor $S_{\mu \nu}^{\rho}=\Gamma_{[\mu \nu]}^{\rho}$, contains the electromagnetic field:

$$
S_{\mu \nu}^{\rho}=k F_{\mu \nu: \sigma} g^{\rho \sigma} .
$$

The trace of the torsion tensor, the torsion vector $S_{\mu}=S_{\mu \nu}^{\nu}$, is thus

$$
S_{\mu}=k F_{\mu}{ }^{\nu}: \nu .
$$

Imposing vanishing of the torsion vector,

$$
S_{\mu}=0,
$$

yields the sourceless Maxwell equations: $F_{\mu}{ }^{\nu}{ }_{\nu}=0$. The nonmetricity tensor $N_{\mu \nu \rho}=$ $g_{\mu \nu ; \rho}[2,16] \pm$ where the semicolon denotes the covariant differentiation with respect to $\Gamma_{\mu \nu}^{\rho}$, is given by

$$
N_{\mu \nu \rho}=k\left(F_{\rho \mu: \nu}+F_{\rho \nu: \mu}\right) .
$$

Eisenhart's affine connection (11) gives the curvature tensor $R_{\mu \sigma \nu}^{\rho}=\Gamma_{\mu \nu, \sigma}^{\rho}-\Gamma_{\mu \sigma, \nu}^{\rho}+$ $\Gamma_{\mu \nu}^{\kappa} \Gamma_{\kappa \sigma}^{\rho}-\Gamma_{\mu \sigma}^{\kappa} \Gamma_{\kappa \nu}^{\rho}[2,[16]$ :

$$
R_{\mu \sigma \nu}^{\rho}=K_{\mu \sigma \nu}^{\rho}+k\left(F_{\mu \nu}: \rho{ }_{\sigma}-F_{\mu \sigma}: \rho\right)+k^{2}\left(F_{\mu \nu}: \kappa F_{\kappa \sigma}: \rho-F_{\mu \sigma}: \kappa F_{\kappa \nu}: \rho\right),
$$

the Ricci tensor $R_{\mu \nu}=R_{\mu \rho \nu}^{\rho}$ :

$$
R_{\mu \nu}=K_{\mu \nu}+k F_{\mu \nu: \rho}^{\rho}+k^{2} F_{\mu}^{\rho}: \sigma F_{\nu: \rho}^{\sigma},
$$

and the curvature scalar $R=R_{\mu \nu} g^{\mu \nu}$ :

$$
R=K+k^{2} F_{\mu: \rho}^{\nu} F_{: \nu}^{\mu \rho},
$$

where $K_{\mu \sigma \nu}^{\rho}, K_{\mu \nu}$ and $K$ are the corresponding Riemannian tensors. The skewsymmetric tensor of homothetic curvature $Q_{\mu \nu}=R_{\rho \mu \nu}^{\rho}=\Gamma_{\rho \nu, \mu}^{\rho}-\Gamma_{\rho \mu, \nu}^{\rho}$, which is proportional to the curl of the trace of the nonmetricity tensor: $Q_{\mu \nu}=-\frac{1}{2}\left(N_{\rho \nu, \mu}^{\rho}-\right.$ $N_{\rho \mu, \nu}^{\rho}$ ), vanishes because of Eqs. (44) and (5). As the gravitational field equation in vacuum, Eisenhart chose vanishing of the symmetrized Ricci tensor [2]:

$$
R_{(\mu \nu)}=0,
$$

$\dagger$ Eisenhart chose the units such that $k=1$.

$\ddagger$ The affine connection is completely determined by the Christoffel symbols, torsion and nonmetricity [12, 13]: $\Gamma_{\mu \nu}^{\rho}=\left\{\begin{array}{c}\rho \\ \mu \nu\end{array}\right\}+S_{\mu \nu}^{\rho}+2 S_{(\mu \nu)}^{\rho}+\frac{1}{2} N_{\mu \nu}^{\rho}-N_{(\mu \nu)}^{\rho}$. 
or

$$
K_{\mu \nu}=-k^{2} F_{\mu: \sigma}^{\rho} F_{\nu: \rho}^{\sigma}
$$

which differs from Einstein's $K_{\mu \nu}=0$. Equations (11), (41) and (9) are the field equations of this theory, giving the metric tensor as a function of the coordinates.

The Lorentz equation of motion in Eisenhart's theory results from the condition [2]:

$$
g_{\mu \nu} u^{\mu} u^{\nu}=\text { const, }
$$

where $u^{\mu}=\frac{d x^{\mu}}{d s}$ is the four-velocity vector tangent to a world line parametrized by $s$, $x^{\mu}=x^{\mu}(s)$. Differentiating Eq. (11) gives

$$
g_{\mu \nu}\left(\frac{d u^{\mu}}{d s}+\left\{\begin{array}{c}
\mu \\
\rho \sigma
\end{array}\right\} u^{\rho} u^{\sigma}\right) u^{\nu}=0
$$

This equation is satisfied by

$$
g_{\mu \nu}\left(\frac{d u^{\mu}}{d s}+\left\{\begin{array}{c}
\mu \\
\rho \sigma
\end{array}\right\} u^{\rho} u^{\sigma}\right)=a_{\nu \rho} u^{\rho}
$$

where $a_{\nu \rho}$ is an arbitrary skewsymmetric tensor. The simplest geometrical choice for $a_{\mu \nu}$ is to identify it with the electromagnetic field tensor $F_{\mu \nu}$ (which is a part of the affine connection (1)):

$$
a_{\mu \nu}=b F_{\mu \nu}
$$

where $b$ is a physical constant. If $b=\frac{e}{m}$, we obtain the classical Lorentz equation for a particle with mass $m$ and electric charge $e$.

\section{Metric-affine gravity}

A general metric-affine Lagrangian density $\mathcal{L}$ depends on the affine connection $\Gamma_{\mu \nu}^{\rho}$ and the curvature tensor, $R_{\mu \sigma \nu}^{\rho}$, as well as the metric tensor $g_{\mu \nu}$ with the Lorentzian signature $(+,-,-,-)$. The simplest metric-affine Lagrangian density that depends on the curvature is the Einstein-Hilbert Lagrangian density for the gravitational field [11]:

$$
\mathcal{L}_{g}=-\frac{1}{2 \kappa} R_{\mu \nu} \mathrm{g}^{\mu \nu}
$$

where $\kappa=8 \pi G(c=1), \mathrm{g}^{\mu \nu}=\sqrt{-g} g^{\mu \nu}$ is the fundamental metric density [6], and $g=\operatorname{det}\left(g_{\mu \nu}\right)$. The total Lagrangian density for the gravitational field and of matter is given by $\mathcal{L}=\mathcal{L}_{g}+\mathcal{L}_{m}$, where the Lagrangian density for matter $\mathcal{L}_{m}$ depends in general on both the metric and connection. The variations of $\mathcal{L}_{m}$ with respect to the metric and connection define, respectively, the dynamical energy-momentum tensor

$$
T_{\mu \nu}=\frac{2}{\sqrt{-g}} \frac{\delta \mathcal{L}_{m}}{\delta g^{\mu \nu}}
$$

and the hypermomentum density [14]:

$$
\Pi_{\rho}^{\mu \nu}=-2 \kappa \frac{\delta \mathcal{L}_{m}}{\delta \Gamma_{\mu \nu}^{\rho}},
$$


which has the same dimension as the connection.

The metric-affine theory based on the Lagrangian density $\mathcal{L}_{g}$ does not determine the connection uniquely because $\mathcal{L}_{g}(15)$ depends only on the symmetric part $R_{(\mu \nu)}$ of the Ricci tensor and thus is invariant under projective transformations [14, 17]:

$$
\Gamma_{\mu \nu}^{\rho} \rightarrow \Gamma_{\mu \nu}^{\rho}+\delta_{\mu}^{\rho} V_{\nu}
$$

where $V_{\nu}$ is a vector function of the coordinates. The same problem occurs if we add to $\mathcal{L}_{g}$ a Lagrangian for matter $\mathcal{L}_{m}$ that does not depend on the connection, for example, representing the electromagnetic field or an ideal fluid. Therefore at least four degrees of freedom must be constrained to make such a theory consistent from a physical point of view [14]. If, however, $\mathcal{L}_{m}$ does depend on the connection, for example, for spinor fields, the projective invariance of the total Lagrangian density $\mathcal{L}=\mathcal{L}_{g}+\mathcal{L}_{m}$ imposes four algebraic constraints on $\Pi^{\mu}{ }_{\rho}^{\nu}$ (cf. Eq. (22) ) and restricts forms of matter that can be described by metric-affine gravity [14]. This restriction has usually the form of a field equation, for example, including the term proportional to $\sqrt{-g} Q_{\mu \nu} Q^{\mu \nu}$ which has the form of the Maxwell Lagrangian for the electromagnetic field, gives the Maxwell-like equations for the tensor $Q_{\mu \nu}$ [17.

From the stationarity of the action $S=\int d^{4} x \mathcal{L}$ under arbitrary variations of $g^{\mu \nu}$ : $\delta S=0$, we obtain the metric-affine Einstein equations:

$$
R_{(\mu \nu)}-\frac{1}{2} R g_{\mu \nu}=\kappa T_{\mu \nu}
$$

The variation of the action for the Lagrangian density (15) with respect to the connection is: $\delta S_{g}=-\frac{1}{2 \kappa} \int d^{4} x \mathrm{~g}^{\mu \nu} \delta R_{\mu \nu}$. Using the Palatini formula for the variation of the Ricci tensor [6, 16]: $\delta R_{\mu \nu}=\delta \Gamma_{\mu \nu ; \rho}^{\rho}-\delta \Gamma_{\mu \rho ; \nu}^{\rho}-2 S_{\rho \nu}^{\sigma} \delta \Gamma_{\mu \sigma}^{\rho}$, we find

$$
\delta S=-\frac{1}{2 \kappa} \int d^{4} x\left(\mathrm{~g}^{\mu \nu}\left(\delta \Gamma_{\mu \nu ; \rho}^{\rho}-\delta \Gamma_{\mu \rho ; \nu}^{\rho}-2 S_{\rho \nu}^{\sigma} \delta \Gamma_{\mu \sigma}^{\rho}\right)+\Pi_{\rho}^{\mu \nu} \delta \Gamma_{\mu \nu}^{\rho}\right) .
$$

Integrating by parts and using the identity $\int d^{4} x\left(\mathrm{~V}^{\mu}\right)_{; \mu}=2 \int d^{4} x S_{\mu} \mathrm{V}^{\mu}$, where $\mathrm{V}^{\mu}$ is an arbitrary vector density, from the stationarity of the action under arbitrary variations of $\Gamma_{\mu \nu}^{\rho}$ we obtain

$$
\mathrm{g}_{; \rho}^{\mu \nu}-\mathrm{g}_{; \sigma}^{\mu \sigma} \delta_{\rho}^{\nu}-2 \mathrm{~g}^{\mu \nu} S_{\rho}+2 \mathrm{~g}^{\mu \sigma} S_{\sigma} \delta_{\rho}^{\nu}+2 \mathrm{~g}^{\mu \sigma} S_{\rho \sigma}^{\nu}=\Pi_{\rho}^{\mu \nu} .
$$

Contracting the indices $\mu$ and $\rho$ in Eq. (21) gives

$$
\Pi_{\sigma}^{\sigma \nu}=0,
$$

which constrains how the connection $\Gamma_{\mu \nu}^{\rho}$ can enter the metric-affine Lagrangian density for matter $\mathcal{L}_{m}$. Equation (21) is equivalent to

$$
\mathrm{g}_{, \rho}^{\mu \nu}+{ }^{*} \Gamma_{\sigma \rho}^{\mu} \mathrm{g}^{\sigma \nu}+{ }^{*} \Gamma_{\rho \sigma}^{\nu} \mathrm{g}^{\mu \sigma}-{ }^{*} \Gamma_{\sigma \rho}^{\sigma} \mathrm{g}^{\mu \nu}=\Pi_{\rho}^{\mu \nu}-\frac{1}{3} \Pi_{\sigma}^{\mu}{ }_{\sigma}^{\sigma} \delta_{\rho}^{\nu},
$$

where ${ }^{*} \Gamma_{\mu \nu}^{\rho}=\Gamma_{\mu \nu}^{\rho}+\frac{2}{3} \delta_{\mu}^{\rho} S_{\nu}$ is the projectively invariant part of the connection (Schrödinger's star-affinity) [6].

$\S$ The variational derivative of a function $\mathcal{L}\left(\phi, \phi_{, \mu}\right)$ with respect to a variable $\phi$ is defined as $\left.\frac{\delta \mathcal{L}}{\delta \phi}=\frac{\partial \mathcal{L}}{\partial \phi}-\left(\frac{\partial \mathcal{L}}{\partial \phi, \mu}\right)_{, \mu}\right)$. 
Equation (23) gives a linear relation between ${ }^{*} \Gamma_{\mu \nu}^{\rho}$ and the hypermomentum density $\Pi_{\rho}^{\mu}{ }_{\rho}^{\nu}$. If we decompose the star-affinity ${ }^{*} \Gamma_{\mu \nu}^{\rho}$ as

$$
{ }^{*} \Gamma_{\mu \nu}^{\rho}=\left\{\begin{array}{c}
\rho \\
\mu \nu
\end{array}\right\}+V_{\mu \nu}^{\rho}
$$

where $V_{\mu \nu}^{\rho}$ is a projectively invariant deflection tensor, then $V_{\mu \nu}^{\rho}$ is linear in $\Pi_{\rho}^{\mu}{ }_{\rho}^{\nu}$ :

$$
V_{\sigma \rho}^{\mu} \mathrm{g}^{\sigma \nu}+V_{\rho \sigma}^{\nu} \mathrm{g}^{\mu \sigma}-V_{\sigma \rho}^{\sigma} \mathrm{g}^{\mu \nu}=\Pi_{\rho}^{\mu \nu}-\frac{1}{3} \Pi_{\sigma}^{\mu \sigma} \delta_{\rho}^{\nu}
$$

The solution of Eq. (25) is given by (cf. [18]):

$$
\begin{aligned}
& V_{\mu \nu}^{\rho}=\frac{1}{2 \sqrt{-g}}\left(\Delta_{\nu}^{\rho}{ }_{\nu}{ }^{\sigma} g_{\mu \sigma}+\Delta_{\mu}^{\rho}{ }_{\mu} g_{\nu \sigma}-\Delta_{\gamma}^{\alpha \beta} g_{\mu \alpha} g_{\nu \beta} g^{\rho \gamma}\right. \\
& \left.+\Omega_{\nu}{ }^{\rho \sigma} g_{\mu \sigma}-\Omega_{\mu}{ }^{\rho \sigma} g_{\nu \sigma}-\Omega_{\gamma}{ }^{\alpha \beta} g_{\mu \alpha} g_{\nu \beta} g^{\rho \gamma}\right),
\end{aligned}
$$

where:

$$
\begin{aligned}
& \Delta_{\rho}^{\mu \nu}=\Sigma_{\rho}^{\mu \nu}-\frac{1}{2} \sum_{\rho}^{\alpha \beta} g_{\alpha \beta} g^{\mu \nu}, \\
& \Sigma_{\rho}^{\mu \nu}=\Pi_{\rho}^{(\mu \nu)}-\frac{1}{3} \delta_{\rho}^{(\mu} \Pi_{\sigma}^{\nu)}, \\
& \Omega_{\rho}^{\mu \nu}=\Pi_{\rho}^{[\mu \nu]}+\frac{1}{3} \delta_{\rho}^{[\mu} \Pi_{\sigma}^{\nu]} .
\end{aligned}
$$

For the connection given by Eq. (24), the Ricci tensor is quadratic in $V_{\mu \nu}^{\rho}$ [16], that is, in $\prod_{\rho}^{\mu \nu}$ :

$$
R_{\mu \nu}=K_{\mu \nu}-\frac{4}{3} S_{[\nu: \mu]}+2 V_{\mu[\nu: \rho]}^{\rho}+V_{\mu \nu}^{\sigma} V_{\sigma \rho}^{\rho}-V_{\mu \rho}^{\sigma} V_{\sigma \nu}^{\rho}
$$

Substituting this $R_{\mu \nu}$ to the metric-affine Einstein equations (19) and moving the terms with $V_{\mu \nu}^{\rho}$ to the right-hand side gives the metric Einstein equations. The Ricci scalar is [16]:

$$
R=K+V_{\sigma: \rho}^{\rho \sigma}-V_{\rho}^{\rho \sigma}: \sigma+V_{\lambda}^{\sigma \lambda} V_{\sigma \rho}^{\rho}-V_{\sigma \lambda \rho} V^{\rho \sigma \lambda} .
$$

\section{Variational formulation of Eisenhart's theory}

Comparing Eq. (11) with (24) and using the condition (44) gives

$$
V_{\mu \nu}^{\rho}=k F_{\mu \nu: \sigma} g^{\rho \sigma} \text {. }
$$

Equation (25) gives

$$
\Pi_{\rho}^{\mu \nu}=\sqrt{-g}\left(V_{\rho}^{\mu \nu}+V_{\rho}^{\nu \mu}-V_{\sigma \rho}^{\sigma} g^{\mu \nu}-V_{\sigma}^{\mu} \delta_{\rho}^{\nu}\right) .
$$

Substituting Eq. (32) to (33) gives the hypermomentum density

$$
\Pi_{\rho}^{\mu \nu}=k \sqrt{-g} g^{\mu \alpha} g^{\nu \beta}\left(F_{\rho \alpha: \beta}-F_{\rho \beta: \alpha}\right),
$$

from which it follows that the matter Lagrangian density $\mathcal{L}_{m}$ that generates (34) via Eq. (17) is given by

$$
\mathcal{L}_{m}=-\frac{k}{\kappa} \sqrt{-g} F_{\rho \alpha: \beta} S_{\mu \nu}^{\rho} g^{\mu \alpha} g^{\nu \beta}
$$

$\|$ We use the fact that the variation with respect to $\Gamma_{\mu \nu}^{\rho}$ in metric-affine gravity is equivalent to the variation with respect to $\Gamma_{\mu \nu}^{\rho}-\left\{\begin{array}{c}\rho \\ \mu \nu\end{array}\right\}$ and the covariant derivative : does not depend on $\Gamma_{\mu \nu}^{\rho}-\left\{\begin{array}{c}\rho \\ \mu \nu\end{array}\right\}$. 
This Lagrangian density, however, also generates the energy-momentum tensor via Eq. (16):

$$
T_{\mu \nu}=\frac{k}{\kappa}\left(F_{\rho \alpha: \beta} S^{\rho \alpha \beta} g_{\mu \nu}-2 F_{\rho[\alpha: \mu]} S_{\nu}^{\rho \alpha}-2 F_{\rho[\alpha: \nu]} S_{\mu}^{\rho \alpha}\right),
$$

which is traceless. The total Lagrangian density is equal to

$$
\mathcal{L}=\sqrt{-g}\left(-\frac{1}{2 \kappa} R-\frac{k}{\kappa} F_{\rho \alpha: \beta} S^{\rho \alpha \beta}\right),
$$

and produces the field equations (19) which, using Eqs. (2) and (36), can be written as

$$
R_{(\mu \nu)}=k^{2}\left(F_{\alpha: \beta}^{\rho} F_{: \rho}^{\alpha \beta} g_{\mu \nu}-2 F_{[\alpha: \mu]}^{\rho} F_{\nu: \rho}^{\alpha}-2 F_{[\alpha: \nu]}^{\rho} F_{\mu: \rho}^{\alpha}\right),
$$

or, using Eq. (7), as

$$
K_{\mu \nu}=k^{2}\left(F_{\alpha: \beta}^{\rho} F_{: \rho}^{\alpha \beta} g_{\mu \nu}-2 F_{[\alpha: \mu]}^{\rho} F_{\nu: \rho}^{\alpha}-2 F_{[\alpha: \nu]}^{\rho} F_{\mu: \rho}^{\alpha}-F_{\mu}{ }^{\rho}: \sigma F_{\nu: \rho}^{\sigma}\right) .
$$

We can obtain a simpler field equation if we add to the right-hand side of Eq. (37) a term: $\tilde{\mathcal{L}}_{m}=\frac{k^{2}}{\kappa} F_{\alpha: \beta}^{\rho} F_{: \rho}^{\alpha \beta}$, so the total Lagrangian density is

$$
\mathcal{L}=\sqrt{-g}\left(-\frac{1}{2 \kappa} R-\frac{k}{\kappa} F_{\rho \alpha: \beta} S^{\rho \alpha \beta}+\frac{k^{2}}{\kappa} F_{\alpha: \beta}^{\rho} F_{: \rho}^{\alpha \beta}\right) .
$$

Equation (34) does not change (cf. footnote 4), while the variation of the action corresponding to the Lagrangian density (40) with respect to $g_{\mu \nu}$ and using Eq. (2) gives

$$
R_{(\mu \nu)}=k_{:(\mu}^{2}\left(2 F_{\nu) \alpha: \beta}^{\alpha \beta}-F_{\alpha: \beta}^{\rho} F_{: \rho}^{\alpha \beta} g_{\mu \nu}\right),
$$

or

$$
K_{\mu \nu}=k^{2}\left(2 F_{:(\mu}^{\alpha \beta} F_{\nu) \alpha: \beta}-F_{\alpha: \beta}^{\rho} F_{: \rho}^{\alpha \beta} g_{\mu \nu}-F_{\mu: \sigma}^{\rho} F_{\nu: \rho}^{\sigma}\right) .
$$

In order to reproduce Eisenhart's equation (10), we should add to the right-hand side of Eq. (37) a term that does not depend on $\Gamma_{\mu \nu}^{\rho}-\left\{\begin{array}{c}\rho \\ \mu \nu\end{array}\right\}$ and that generates, via Eq. (16)), the energy-momentum tensor $-\frac{k^{2}}{\kappa}\left(F_{\alpha: \beta}^{\rho} F_{: \rho}^{\alpha \beta} g_{\mu \nu}-2 F_{[\alpha: \mu]}^{\rho} F_{\nu: \rho}^{\alpha}-2 F_{[\alpha: \nu]}^{\rho} F_{\mu: \rho}^{\alpha}\right)$ which cancels the energy-momentum tensor (36).

The problem with Eisenhart's field equation (10) and its modifications (39) and (42) occurs when we apply to them the contracted Bianchi identity $\left(K_{\mu}{ }^{\nu}-\frac{1}{2} K \delta_{\mu}^{\nu}\right)_{: \nu}=0$. This identity gives additional constraints on the electromagnetic field tensor, which is inconsistent with the Maxwell electrodynamics. Therefore we must add to the righthand side of Eq. (37) a term that does not depend on $\Gamma_{\mu \nu}^{\rho}-\left\{\begin{array}{c}\rho \\ \mu \nu\end{array}\right\}$ and that generates, via Eq. (16) , the energy-momentum tensor $-\frac{k^{2}}{\kappa}\left(F_{\alpha: \beta}^{\rho} F_{: \rho}^{\alpha \beta} g_{\mu \nu}-2 F_{[\alpha: \mu]}^{\rho} F_{\nu: \rho}^{\alpha}-2 F_{[\alpha: \nu]}^{\rho} F_{\mu: \rho}^{\alpha}-\right.$ $\left.F_{\mu}{ }^{\rho}: \sigma F_{\nu: \rho}^{\sigma}\right)$, resulting in Einstein's field equation $K_{\mu \nu}=0$.

The above variational formulation of Eisenhart's theory is not complete because we did not include the condition that the torsion vector $S_{\mu}$ be zero in the Lagrangian.

9 The Lagrangian density (37) is dynamically equivalent to $\mathcal{L}=\sqrt{-g}\left(-\frac{1}{2 \kappa} R+\frac{k}{\kappa} F_{\rho \alpha} S^{\rho \alpha \beta}: \beta\right)$, in which the electromagnetic field tensor couples to the divergence of the torsion tensor $S_{: \rho}^{\mu \nu \rho}$. 
This condition enters the Lagrangian density as a Lagrange-multiplier term $\sqrt{-g} B^{\mu} S_{\mu}$, where $B^{\mu}$ is a vector [13, 18]. The total Lagrangian density (37) becomes

$$
\mathcal{L}=\sqrt{-g}\left(-\frac{1}{2 \kappa} R-\frac{k}{\kappa} F_{\rho \alpha: \beta} S^{\rho \alpha \beta}+B^{\mu} S_{\mu}\right),
$$

Treating the term $\sqrt{-g} B^{\mu} S_{\mu}$ as a matter part of the Lagrangian adds, via Eq. (17), a term $-2 \kappa \sqrt{-g} B^{[\mu} \delta_{\rho}^{\nu]}$ to the hypermomentum density $\Pi^{\mu}{ }_{\rho}^{\nu}$. Since the deflection tensor $V_{\mu \nu}^{\rho}$ is linear in $\Pi_{\rho}^{\mu}{ }_{\rho}^{\nu}$, the corresponding change in $V_{\mu \nu}^{\rho}$ is the solution of Eq. (26) for $\Pi_{\rho}^{\mu}{ }^{\nu}=-2 \kappa \sqrt{-g} B^{[\mu} \delta_{\rho}^{\nu]}$, that is:

$$
V_{\mu \nu}^{\rho}=\frac{\kappa}{4}\left(3 B^{\rho} g_{\mu \nu}+B_{\mu} \delta_{\nu}^{\rho}-3 B_{\nu} \delta_{\mu}^{\rho}\right) .
$$

The total deflection tensor is

$$
V_{\mu \nu}^{\rho}=k F_{\mu \nu: \sigma} g^{\rho \sigma}+\frac{\kappa}{4}\left(3 B^{\rho} g_{\mu \nu}+B_{\mu} \delta_{\nu}^{\rho}-3 B_{\nu} \delta_{\mu}^{\rho}\right),
$$

which gives, using Eq. (24), the torsion vector

$$
S_{\mu}=-k F_{\mu: \nu}^{\nu}+\frac{3 \kappa}{2} B_{\mu} .
$$

Varying the action corresponding to (43) with respect to $B^{\mu}$ gives $S_{\mu}=0$ which results in

$$
B^{\mu}=\frac{2 k}{3 \kappa} j^{\mu},
$$

where $j^{\mu}=F_{: \nu}^{\nu \mu}$ is the conserved $\left({j^{\mu}}_{: \mu}=0\right)$ electromagnetic current vector. Therefore the variational formulation of Eisenhart's unified theory generalizes it to electromagnetic fields with sources, represented by a Lagrange-multiplier constraint in the action imposing a traceless torsion.

\section{References}

[1] Goenner H F M 2004 Liv. Rev. Rel. 72

[2] Eisenhart L P 1927 Non-Riemannian Geometry (New York: Amer. Math. Soc.)

[3] Weyl H 1918 Sitzungsber. Preuss. Akad. Wiss. (Berlin) 465 Weyl H 1922 Space, Time, Matter (London: Methuen)

[4] Kaluza T 1921 Sitzunsgber. Preuss. Akad. Wiss. (Berlin) 966 Klein O 1926 Z. Phys. 37895

Einstein A and Bergmann P 1938 Ann. Math. 39683

[5] Einstein A and Straus E G 1946 Ann. Math. 47731 Schrödinger E 1947 Proc. R. Ir. Acad. A 51147 Einstein A 1948 Rev. Mod. Phys. 2035

[6] Schrödinger E 1950 Space-Time Structure (Cambridge: Cambridge Univ. Press)

[7] Cartan É 1922 Compt. Rend. Acad. Sci. 174593

[8] Lord E A 1976 Tensors, Relativity and Cosmology (New Delhi: McGraw-Hill) Macias A and Dehnen H 1991 Class. Quantum Grav. 8203

[9] Eisenhart L P 1956 Proc. Natl. Acad. Sci. USA 42249

[10] Eisenhart L P 1956 Proc. Natl. Acad. Sci. USA 42646

Eisenhart L P 1956 Proc. Natl. Acad. Sci. USA 42878

Eisenhart L P 1957 Proc. Natl. Acad. Sci. USA 43333 
[11] Einstein A 1925 Sitzungsber. Preuss. Akad. Wiss. (Berlin) 414

Einstein A and Kaufman B 1955 Ann. Math. 62128

[12] Smalley L L 1977 Phys. Lett. A 61436

[13] Hehl F W and Kerlick G D 1978 Gen. Rel. Grav. 9691

[14] Sandberg V D 1975 Phys. Rev. D 123013

Hehl F W, von der Heyde P, Kerlick G D and Nester J M 1976 Rev. Mod. Phys. 48393

Hehl F W, Lord E A and Smalley L L 1981 Gen. Rel. Grav. 131037

Hehl F W, McCrea J D, Mielke E W and Ne'eman Y 1995 Phys. Rep. 2581

[15] Ferraris M and Kijowski J 1982 Gen. Rel. Grav. 14165

[16] Schouten J A 1954 Ricci-Calculus (New York: Springer-Verlag)

[17] Ponomarev V N and Obukhov Y N 1982 Gen. Rel. Grav. 14309

[18] Popławski N J 2007 Ann. Fond. L. de Broglie 32335 